\title{
Photoresponsive Liquid-Crystalline Polymer Films Bilayered with an Inverse Opal Structure
}

\author{
Norihisa Akamatsu $^{1}$, Miho Aizawa ${ }^{1}$, Ryoichi Tatsumi ${ }^{1}$, Kyohei Hisano ${ }^{1}$, Arri Priimagi ${ }^{1,2}$, \\ Atsushi Shishido ${ }^{1,3 *}$ \\ ${ }^{1}$ Laboratory for Chemistry and Life Science, Tokyo Institute of Technology, \\ R1-12, 4259 Nagatsuta, Midori-ku, Yokohama 226-8503, Japan \\ ${ }^{2}$ Department of Chemistry and Bioengineering, Tampere University of Technology, \\ Korkeakoulunkatu 8, Tampere, 33720, Finland \\ ${ }^{3}$ PRESTO, JST, 4-1-8 Honcho, Kawaguchi 332-0012, Japan
}

Keywords: Photoresponsive film, Inverse opal structure, Bragg diffraction

\section{Introduction}

Tunable photonic crystal is an area of the recent intense interest, in which Bragg diffraction peak can be controlled by external stimuli $[1,2]$. The simplest photonic crystal is fabricated by the close-packing of spheres, similar to that which forms opals in nature [3,4]. Furthermore, an inverse opal structure can be created by removing sphere after injection of other materials into the gap of opal structure. This structure containing porous lattice has the same properties, forms freestanding film, and is expected as tunable photonic crystals $[5,6]$.

Azobenzene, which is known as one of the most popular photochromic molecules, reversibly changes its conformation between trans and cis isomers by photoisomerization and thermal isomerization. As with many applications of light-reversible phenomena, there is great potential for azobenzene materials due to their superior speed, reversibility, and tunability [7]. Asher and coworkers developed photoresponsive photonic crystal using azobenzene [8]. When a gel containing azobenzene was irradiated with UV light, Bragg peak was reversibly shifted about $60 \mathrm{~nm}$ by swelling and deswelling of the gel. Takeoka and coworkers prepared inverse opal structure into the gel with azobenzene, and found that the shift of Bragg peak can be induced by photoisomerization of azobenzene by photoirradiation [9]. However, these preparation processes are complex and the obtained films require organic solvent to control the reflection spectra. If the solvent-free photoresponsive photonic crystal can be created, there is possibility of using for enlarging applications.

Due to the photodeformation of azobenzene moieties, reversible photoinduced motion of crosslinked liquid-crystalline polymers (CLCP) containing azobenzene has been realized. LC materials have drawn a great deal of attention due to the capability for tuning the band structure through external stimuli [10]. The CLCP films with an azobenzene reversibly expanded and contracted by UV and Vis irradiations [11,12]. Particularly, surface specific contraction in azobenzene-containing LC films brought about photoinduced bending [13].

In our previous work, we prepared azobenzene-containing CLCP films with bilayer structure by a simple technique [14]. The bilayer film showed photodeformation by irradiation of UV light similar to the single layer film. If the inverse opal structure is introduced to photodeformable materials, it is possible to form the solvent-free phototunable photonic crystals.

In this study, an inverse opal film was layered to a photodeformable film, and its photoresponsive behavior was investigated. This bilayer films composed of colorless photonic crystal using inverse opal structures and colored photoresponsive layers with azobenzene-containing CLCP. 


\section{Experimental}

We prepared bilayer films with an azobenzene-containing photoresponsive layer and an inverse opal layer. The chemical structures of the compounds used are shown in Fig. 1. The constituent compounds were synthesized according to previously reported method [15]. The photoresponsive layer was prepared according to published procedures [14]. The mixture of DA9AB, A6BZ6, and C6A with the molar ratio of 20:40:40 containing $1 \mathrm{~mol} \%$ photoinitiator (Ciba Specialty, Irgacure 784) was injected into the $20 \mu \mathrm{m}$ thick glass cell at $90{ }^{\circ} \mathrm{C}$ (in an isotropic phase). The glass cell was coated with rubbed polyimide (JSR, AL1254) in order to obtain homogeneous alignment of mesogens. The mixture was slowly cooled to an LC temperature at $80{ }^{\circ} \mathrm{C}$ at a rate of $0.5{ }^{\circ} \mathrm{C} / \mathrm{min}$. Photopolymerization using a $500-\mathrm{W}$ high-pressure mercury lamp (Ushio, SX-UI501HQ) through glass filters (AGC techno glass, Y-52 and IRA-25S) was carried out for $2 \mathrm{~h}$ at wavelengths over $540 \mathrm{~nm}$ at the light intensity of $4 \mathrm{~mW} / \mathrm{cm}^{2}$. After the photopolymerization, the glass cell was opened, and the film on a glass substrate was obtained as the photoresponsive layer.
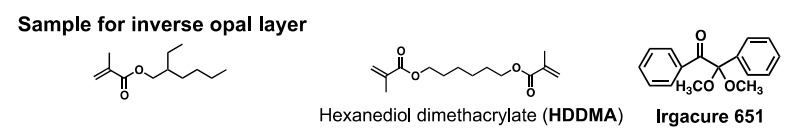

Sample for photoresponsive layer
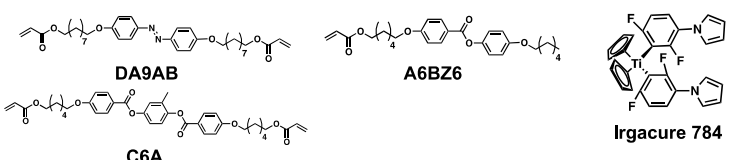

Fig. 1. Chemical structures of the compounds used in this study.

Fig. 2 illustrates the fabrication process of the bilayer inverse opal film. We first prepared the colloid crystal on a glass substrate. Silica particles were synthesized through sol-gel chemistry by the Stöber method and then the colloid crystal was fabricated by dropping the silica dispersion onto a clean glass substrate [16]. The glass cell was prepared with two glass substrates on the photoresponsive layer and colloid crystal layer by stacking them together with spacer of $120 \mu \mathrm{m}$ thickness. The mixture of EHM and HDDMA with the molar ratio of $85: 15$ containing 1 mol\% photoinitiator (Ciba Specialty, Irgacure 651) was injected into the cell at room temperature. Photopolymerization was conducted at 366-nm UV light at the light intensity of $0.4 \mathrm{~mW} / \mathrm{cm}^{2}$ with a high-pressure mercury lamp equipped with glass filters (AGC techno glass, IRA-25S, UV-35, UV-D36-A) and polarized filter (SIGMAKOKI, SPF-50C-32) for $1 \mathrm{~h}$. Polarization direction of the polarized light was set perpendicular to the molecular alignment direction of the photoresponsive layer. Then, the polymerized bilayered film with an opal structure was taken out from the glass cell. Finally, the bilayer opal film was immersed in $5 \mathrm{wt} \%$ hydrofluoric acid solution for $1 \mathrm{~h}$ to remove the silica colloids. After that, we obtained the free-standing bilayer inverse opal film.

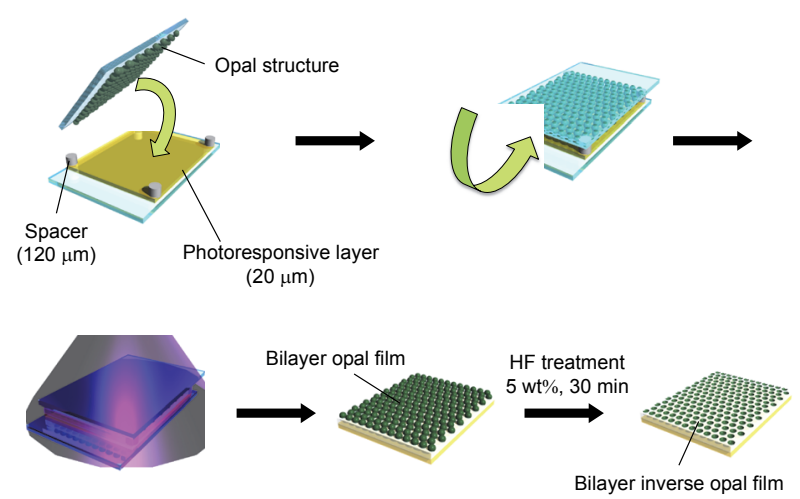

Fig. 2. Preparation of bilayer inverse opal films.

\section{Results and Discussion}

SEM images of the obtained bilayer opal film and bilayer inverse opal film are shown in Fig. 3. It has been found that the silica nanoparticles in the bilayer opal films periodically arranged and their mean diameter is $298 \mathrm{~nm}$. Fig. $3 \mathrm{~b}$ shows that after HF treatment, porous structures are formed by removal of silica colloids. This result means that the periodic structure derived from the face-centered cubic opal structure could be maintained in the process of photopolymerization and removal of nanoparticles. The diameter of porous structures measured by SEM image was $301 \mathrm{~nm}$, which is similar to the diameter of the original opal film. Fig. $3 \mathrm{c}$ and $3 \mathrm{~d}$ show photographs of bilayer inverse opal film. In the photoresponsive layer, the film was colored due to azobenzene. On the other hand, in the inverse opal layer, the film looked completely different; red color based on azobenzene was not seen but green color was observed according to the structural color of the inverse opal structure. This result revealed that colorless photonic crystal layer was successfully fabricated.

Reflection spectra were measured for the prepared opal structure, bilayer opal film and 
bilayer inverse opal film. As shown in Fig. 4, Bragg reflection peaks due to the periodic structure were confirmed in all samples.

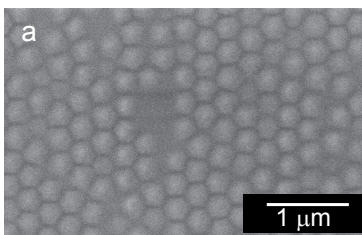

Bilayer opal film

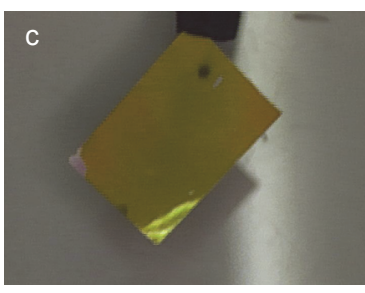

Photoresponsive layer
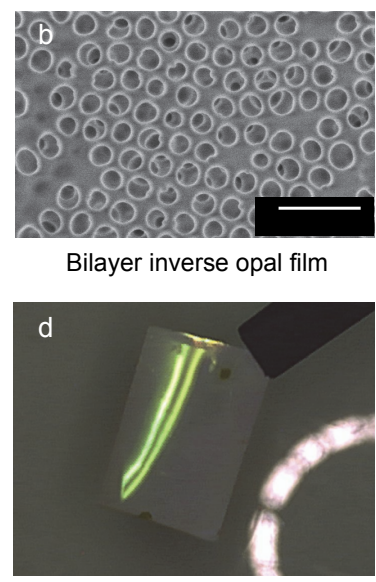

Inverse opal layer
Bilayer inverse opal film

Fig. 3. SEM images of bilayer opal film (a) and bilayer inverse opal film (b). Photographs of bilayer inverse opal film for inverse opal layer side (c) and photoresponsive layer side (d).

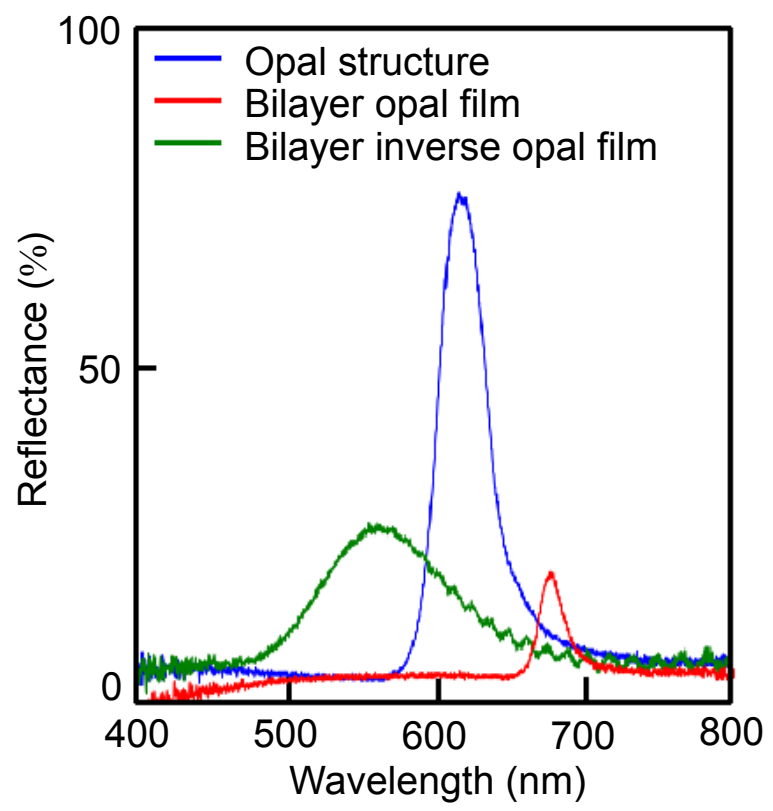

Fig. 4. Reflection spectra of the opal structure (blue), bilayer opal film (red) and bilayer inverse opal film (green).

In the reflection spectra of the colloid crystal and bilayer inverse opal film, the intense and broad peaks appeared at $619 \mathrm{~nm}$ and $555 \mathrm{~nm}$, respectively. These Bragg reflection peaks of the structural color provide us with periodicity of the structure. The reflection peak wavelength $\lambda$ is described by the following equation (1),

$$
\lambda_{\max }=2 \sqrt{\frac{2}{3}} d \sqrt{\sum_{i} n_{i}^{2} \phi_{i}-\sin ^{2} \theta}
$$

where $\mathrm{n}$ is the refractive index, $\boldsymbol{\theta}$ is the incident angle, and $\varphi$ is the volume fraction. Based on the Bragg's equation, the particle diameter $\boldsymbol{d}$ is given by obtained reflection peak $\lambda_{\max }$. Table 1 shows the result of calculated particle dimater $\boldsymbol{d}$, where we use $\boldsymbol{\theta}=0^{\circ}, \boldsymbol{n}_{\text {air }}=1.00 . \boldsymbol{n}_{\mathrm{SiO}_{2}}=1.40, \boldsymbol{n}_{\text {polymer }}=1.48$.

Table 1. Peak wavelength and distance between particles in prepared periodic structures.

\begin{tabular}{ccccc}
\hline & $\Lambda_{\max }(\mathrm{nm})$ & Reflectance $(\%)$ & $d_{\text {Bragg }}(\mathrm{nm})$ & $d_{\text {SEM }}(\mathrm{nm})$ \\
\hline Opal structure & 619 & 83 & 289 & 290 \\
Bilayer opal film & 680 & 20 & 293 & 298 \\
Bilayer inverse opal film & 555 & 30 & 299 & 301 \\
\hline
\end{tabular}

The refractive index of polymer was measured using Abbe refractometer. Because obtained films formed the face-centered cubic structure, volume fraction $\varphi$ of lattice and interspace was defined as 0.741 and 0.259 , respectively. It has been revealed that the particle diameter $\boldsymbol{d}_{\text {SEM }}$ measured by SEM images and calculated $\boldsymbol{d}_{\text {Bragg }}$ are approximately matched for all films. It clearly demonstrates the reflection peaks appeared by the periodic structures.

Fig. 5 shows the investigation of the reflection peak shift by photodeformation. As shown in Fig. $5 \mathrm{~b}$, the reflection peak was observed at $555 \mathrm{~nm}$ before UV irradiation. When the free-standing bilayer inverse opal film was irradiated with UV light $\left(366 \mathrm{~nm}, 100 \mathrm{~mW} / \mathrm{cm}^{2}\right)$, the film bent because of photoisomerization of azobenzene, and the peak
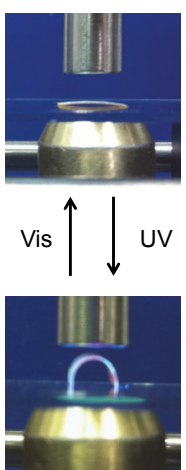

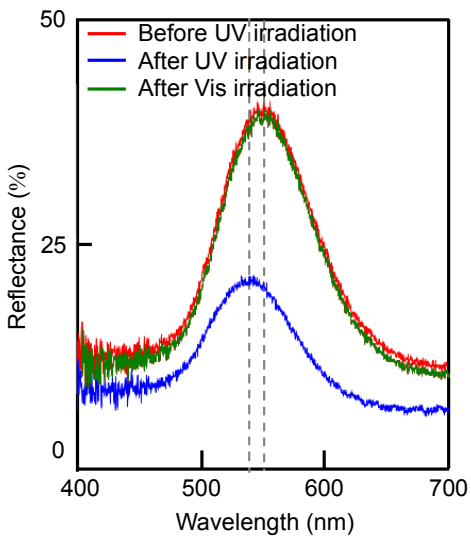

Fig. 5. Photographs (a) and reflection spectra (b) of the photoinduced bending inverse opal film.

wavelength of reflection spectra was changed. During this process, the reflection peak shifted to shorter wavelength, and the peak reached $539 \mathrm{~nm}$, which means the peak was shifted by $16 \mathrm{~nm}$. By 
exposure of the film to Vis light $(530 \mathrm{~nm}, 30$ $\mathrm{mW} / \mathrm{cm}^{2}$ ), the bent film reverted to the original shape and the reflection peak wavelength also recovered to $555 \mathrm{~nm}$, which is the initial peak wavelength. The obtained film showed good repeatability of the reflection peak.

\section{Conclusion}

Colorless, solvent-free photoresponsive photonic crystal was fabricated based on a liquid-crystalline polymer film bilayered with an inverse opal structure. The azobenzene-containing photoresponsive layer and photonic crystal layer using inverse opal structure were stuck by photopolymerization process. The reflection spectra of the inversed opal films showed the reflection peak shift induced by the shrinking of the periodicity by photoinduced bending. The repeated reflection peak shift was for the first time achieved in colorless, solvent-free bilayer CLCP films. These unique properties should be useful for the applications such as photonic paper and reflective display.

\section{References}

1. E. Yablonovitch, Phys. Rev. Lett., 58 (1987) 2059.

2. S. John, Phys. Rev. Lett., 58 (1987) 2486.

3. S. H. Im, M. H. Kim, O. O. Park, Chem. Mater., 15 (2003) 1797.

4. Z. Z. Gu, A. Fujishima, O. Sato, Chem. Mater., 14, (2002) 760.
5. K. Matsubara, M. Watanabe, Y. Takeoka, Angew. Chem. Int. Ed., 46 (2007) 1688.

6. J. Zhao, Y. Liu, Y. Yu, J. Mater. Chem. C, 2 (2014) 10262.

7. A. Priimagi, C. Barrett, A. Shishido, J. Mater. Chem. C, 2 (2014) 7155; A. Shishido, Polym. J. 42 (2010) 525.

8. M. Kamenjicki, I. K. Lednev, A. Mikhonin, R. Kesavamoorthy, S. A. Asher, Adv. Funct. Mater., 13, (2003) 774.

9. K. Matsubara, M. Watanabe, Y. Takeoka, Angew. Chem. Int. Ed., 46 (2007) 1688.

10. T. Kato, N. Mizoshita, K. Kishimoto, Angew. Chem., Int. Ed., 45 (2006) 38.

11. M. Yamada, M. Kondo, J. Mamiya, Y. Yu, M. Kinoshita, C. J. Barret, T. Ikeda, Angew. Chem. Int. Ed., 47 (2008) 4986.

12. M. Yamada, M. Kondo, R. Miyasato, Y. Naka, J. Mamiya, M. Kinoshita, A. Shishido, Y. L. Yu, C. J. Barret, T. Ikeda, J. Mater. Chem., 19 (2009) 60.

13. N. Akamatsu, W. Tashiro, K. Saito, J. Mamiya, M. Kinoshita, T. Ikeda, J. Takeya, S. Fujikawa, A. Priimagi and A. Shishido, Sci. Rep., 4 (2014) 5377.

14. R. Tatsumi, J. Mamiya, M. Kinoshita, A. Shishido, Sci. Adv. Mater., 6 (2014) 1432.

15. A. Shimamura, A. Priimagi, J. Mamiya, T. Ikeda, Y. Yu, C. J. Barrett, A. Shishido, $A C S$ Appl. Mater. Interfaces, 3 (2011) 4190.

16. J. H. Zhang, P. Zhan, Z. L. Wang, W. Y. Zhang, N. B. Ming, J. Mater. Res., 18 (2003) 649. 